\title{
ASPECTS CONCERNING OF THE VEHICLE- PEDESTRIAN IMPACT AT LOW Velocities
}

\author{
Soica, A.; Motoc LuCA, D.; LAChe, S. \& TARulescu, S.
}

Abstract: The paper approaches the impact phenomemon between a vehicle and pedeastrians, at low velocities, from multibody approach point of view. The experimental data retreived were used to describe the dynamics and kinematics of the phenomeon that takes places in such cases, and were compared with the national/international data bases.

Key words: automotive, pedestrian, impact, low velocity, multibody
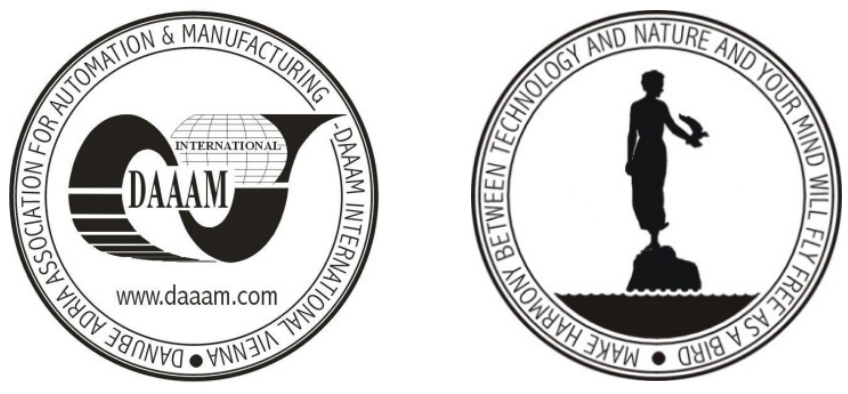

Authors' data: Dr. Soica, A[drian]*; Dr. Ass. Prof. Motoc Luca, D[ana $]^{* *}$; Prof. Dr. Ing. Lache, S[imona]***; Tarulescu, S[telian]*, *Transilvania University of Brasov, Al. Mimozei, nr. 3, bl 79, sc. A, Apt. 16, 500377, Brasov, RO, **Transilvania University of Brasov, 29 Eroilor Av., 500107, Brasov, RO, ***Transilvania University of Brasov, Crisului 6A/8, 500283, Brasov, RO, a.soica@unitbv.ro,danaluca@unitbv.ro,slache@unitbv.ro,rtarulescu@unitbv.ro

This Publication has to be referred as: Soica, A[drian]; Motoc Luca, D[ana]; Lache, S[imona] \& Tarulescu, S[telian] (2008). Aspects Concerning of the VehiclePedestrian Impact at Low Velocities, Chapter 65 in DAAAM International Scientific Book 2008, pp. 797-810, B. Katalinic (Ed.), Published by DAAAM International, ISBN 978-3-901509-66-7, ISSN 1726-9687, Vienna, Austria

DOI: $10.2507 /$ daaam.scibook.2008.65 